\title{
DISEÑO DE UNA ESTACIÓN TERRENA SDR PARA SATÉLITES DE ÓRBITA BAJA
}

\section{Design of a SDR ground station for Low-orbit satellites}

\author{
Jaime Enrique Orduy Rodríguez¹, Iván Felipe Rodríguez Barón², Cristian Andrés Cubillos Chaparro³. \\ ${ }^{1-3}$ Departamento de Ingeniería Aeronáutica, Fundación Universitaria los Libertadores (Bogotá, Colombia) \\ Email: ${ }^{1}$ jeorduyr@libertadores.edu.co, ${ }^{2}$ ifrodriguezb@libertadores.edu.co, \\ ${ }^{3}$ cacubillosc@libertadores.edu.co.
}

(Recibido Agosto 15 de 2019 y aceptado Febrero de 2020)

\section{Resumen}

Los sistemas espaciales se caracterizan por la planeación y creación de todos sus segmentos con el fin de que en conjunto se cumpla una misión; con relación a estos sistemas, en Colombia han sido desarrollados dos misiones espaciales: el Libertad 1, un CubeSat DE 1 U de la Universidad Sergio Arboleda y el FACSAT1, un CubeSat de 3 U de la Fuerza Aérea Colombiana. Dichas misiones generaron el desarrollo completo del segmento espacial denominado satélite, a su vez han generado el segmento terrestre denominado Estación terrena. Para el asegurar el éxito del cumplimiento de la misión se hace necesario tener control sobre el desarrollo del segmento terrestre; es decir, las estaciones terrenas, por esta razón, y con la motivación de la construcción del segmento terrestre de una misión espacial en la Fundación Universitaria Los Libertadores, se logró definir un diseño preliminar para rastrear satélites del tipo pico y nano dentro del segmento radioaficionado o universitario. Con base en la investigación aplicada se buscó la generación de conocimiento dentro de la institución con la aplicación en el sector espacial, desarrollando las pautas necesarias que permitieran la identificación de los diferentes componentes para la construcción de una Estación Terrena para pequeños satélites. Con la búsqueda de las capacidades necesarias para obtener una recepción adecuada de los satélites, se obtuvo una estación terrena de bajo costo usando tecnología comercial y, principalmente, componentes de hardware del tipo SDR. Finalmente, se realizó una comparación con estaciones terrenas bajo costo para mirar la contribución en Colombia.

Palabras clave: estación terrena, CubeSat, satélite, SDR.

\begin{abstract}
Space systems are characterized by the planning and creation of all their segments in order for a mission to be accomplished together; regarding these systems, two space missions have been developed in Colombia: Libertad 1, a $1 \mathrm{U}$ CubeSat from Sergio Arboleda University and FACSAT1, a $3 \mathrm{U}$ CubeSat from the Colombian Air Force. These missions generated the complete development of the space segment called satellite, in turn they have generated the terrestrial segment called ground station. In order to ensure the success of any mission, it is necessary to have control over the development of the ground segment, it does it mean, the ground stations; for this reason, and being motivated in this segment at the Fundación Universitaria Los Libertadores, it was possible to define a preliminary design to track pico and nano satellites within the amateur or university segment. Based on applied research, the generation of knowledge was sought within the institution with its application in the space sector, developing the necessary guidelines that would allow the identification of the different components for the construction of an Earth Station for small satellites. With the search for the necessary capacities to obtain an adequate reception of the satellites, a low-cost earth station was obtained using commercial technology and mainly hardware components of the SDR type. Finally, a comparison was made with low cost earth stations to look at the contribution in Colombia.
\end{abstract}


Key words: ground station, CubeSats, satellite, SDR.

\section{INTRODUCCIÓN}

El objetivo principal de esta investigación se basa en el desarrollo tecnologico del segmento terreste de las misiones espaciales en Colombia, determinando la realización del diseño preliminar para la futura implementación de una Estación Terrena de bajo costo para rastreo de satélites de órbita baja-LEO- en la Fundación Universitaria los Libertadores, que pueda servir de ayuda para la implementación del segmento terrestre en proyectos de pico y nanosatélites en universidades con el propósito de tener autonomía sobre la planificación y ejecución de este segmento, en vez de comprar los equipos y depender tecnológicamente de empresas extranjeras.

El segmento espacial de los sistemas espaciales ya diseñados y activos en el espacio, como los satélites del servicio radioaficionado y del tipo universitarios alrededor del mundo, se pueden comunicar con un segmento terrestre diferente al desarrollado por ellos, esto se logra con la implementación de una estación terrena que satisfaga las necesidades y protocolos de comunicación, con el análisis previo de cada misión. La importancia de desarrollar estas estaciones se centra en la capacitación de la mano de obra y el fortalecimiento de la academia en temas aeroespaciales. De esta forma, se presenta del diseño preliminar de la Estación terrena denominada FULL-ET, basada en el documento proceso de referencia para el desarrollo de la arquitectura de una estación terrena para pico y nanosatélites.

\section{SISTEMA DE ESTACIÓN TERRENA (GSTS)}

Una estación terrena es un conjunto de equipos de comunicaciones y de cómputo que puede ser fijo o móvil [1], las estaciones terrenas usualmente tienen el principal propósito de transmitir, recibir y realizar seguimiento de un satélite y estas son de total importancia para el éxito de una misión debido a que es el primer y el último punto del enlace de comunicación. Además, se pueden establecer, según Ichikawa otras funciones de las estaciones [2], siendo:

Operaciones de telemetría: son utilizadas para la adquisición y registro de datos y estado del satélite.

Operaciones de telecomando: son usadas para la interrogación y control de las funciones del satélite.

Operaciones de control para poder determinar los parámetros orbitales, programar los pasos del satélite y monitorear la carga del computador abordo.

Operaciones de procesamiento de datos para poder presentar los datos científicos e ingenieriles de la forma adecuada para la misión.

Permitir los enlaces de voz y datos a otras estaciones en diferentes partes del mundo. Por otro lado, las estaciones pueden ser clasificadas en de acuerdo con Penna [3] en:

Estación fija: Este tipo de estación se encuentra localizada en un único punto determinado, para ser construida debe tener un previo estudio sobre factores que puedan afectar para la interacción con el satélite, tales como: altura, topografía del lugar (edificios y alteraciones del terreno como montañas), clima y demás circunstancias que puedan ser relevantes para el establecimiento de esta estación.

Estación móvil: son las estaciones que pueden tener comunicación con un satélite en diferentes puntos o estando en movimiento, estas usualmente cuentan con equipamiento básico para su facilidad de traslado las que son llevadas, principalmente, en medios de transporte.

Estación universitaria: estas estaciones básicamente son diseñadas para tener comunicación con satélites desarrollados por estudiantes y, esencialmente, son construidas con servicios de radioaficionados.

Asimismo, las estaciones terrenas universitarias están 
principalmente diseñadas para comunicarse con satélites desarrollados por estudiantes y/o profesores. El tamaño y la complejidad de la estación se establecerán por el servicio que desempeñará y las características operativas requeridas por el segmento espacial. Las estaciones de estos proyectos se construyen con base a los conocimientos técnicos del servicio radioaficionado, realizando la comunicación en las bandas UHF y VHF, reguladas por International Amateur Radio Union (IARU), y utilizando paquetes del protocolo de comunicación AX.25 [4].

La Figura 1 representa, por medio de un diagrama de bloques, los subsistemas de la estación, siendo éstos: el sistema irradiante, el control de la antena, el transceptor de RF, el módem, el software de gestión y la interfaz de usuario, y sus relaciones con los otros segmentos que componen una misión espacial, como el CCS y el segmento espacial.

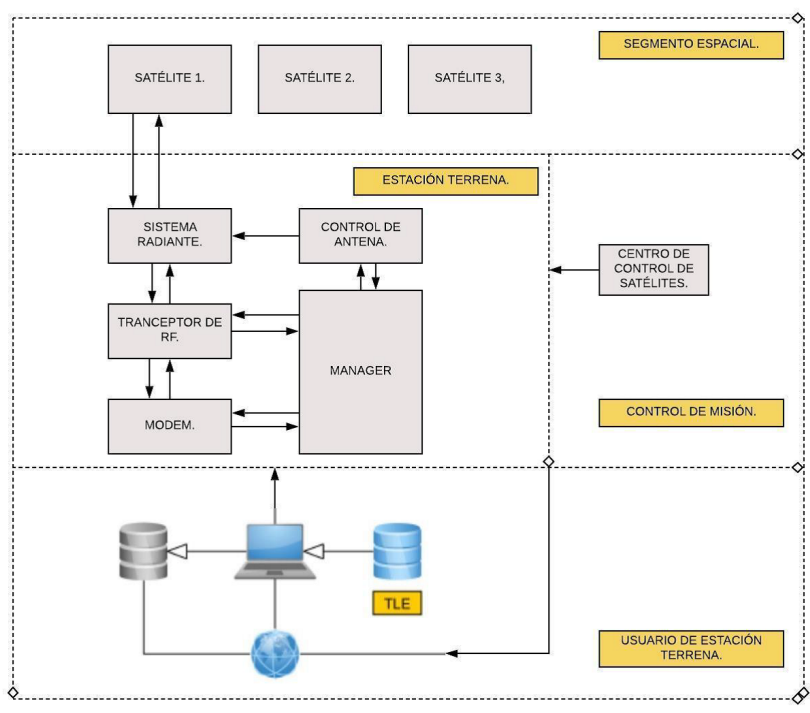

Figura 1. Diagrama de bloques para una estación terrena para pico y nanosatélites [5]

\section{METODOLOGÍA}

De acuerdo con Lozada la investigación aplicada busca la generación de conocimiento con aplicación a los problemas del sector productivo con base en los hallazgos tecnológicos de la investigación básica, ocupándose de la unión entre la teoría y el producto [6]. Con lo anterior, el proyecto se dividió en tres fases:

Fase I: Conceptualización del desarrollo de la estación terrena en la Fundación Universitaria los Libertadores.

Fase II: Evaluación de las necesidades para la implementación de una estación terrena, teniendo en cuenta las características de operación deseadas.

Fase III: Montaje preliminar de la estación terrena y pruebas en el lugar planteado, especificando el tipo de componentes que se implementarán, siempre buscando el menor costo, pero la mejor eficiencia de trabajo.

El diseño preliminar de la Estación Terrena a ser implementada en la Fundación Universitaria los Libertadores está basado en el documento "Processo de referência para o desenvolvimento da arquitetura de uma estação terrena para pico e nanosatélites", el cual presenta un proceso de referencia para la implementación de estaciones terrenas para Picosatélites y Nanosatélites, de acuerdo con las necesidades de la misión, de tal manera que, se pudiera tomar la mejor decisión a la hora de evaluar la planeación y la ejecución del segmento terrestre de proyectos con pequeños satélites.

De acuerdo con Orduy en proyectos de pequeños satélites realizados en Brasil, los equipos de desarrollo de estos proyectos compraron y utilizaron infraestructuras de estaciones terrenas existentes, con el fin de no preocuparse por el desarrollo paralelo de la estación terrena. Esta decisión genera ventajas, ya que ahorra tiempo, recursos humanos y fundamentalmente dinero. Sin embargo, el diseñar la estación terrena junto con el segmento espacial aumenta la capacidad de modificación das arquitecturas física y funcional, ya que previene defectos de compatibilidad entre la estación y el segmento espacial [5].

El diseño de la Estación Terrena a ser implementada en la Fundación Universitaria los Libertadores tendrá en cuenta la flexibilidad de la operación y el bajo costo. Además, el diseño se realiza con un abordaje Bottom-Up o análisis 
ascendente, el cual se desarrolla para describir en detalle el funcionamiento y el comportamiento del sistema. Este enfoque describe el proceso de análisis de un sistema mediante la identificación de sus componentes y sus interrelaciones, así como la creación de una representación del sistema con un alto nivel de abstracción [7].

De igual forma, Orduy establece que la primera etapa del desarrollo de la estación corresponde a la decisión de los parámetros que son necesarios para realizar el cálculo del enlace de comunicaciones, comúnmente denominado Link Budget, ya que dicho cálculo de enlace tiene como objetivo definir las características de los componentes de la estación. En principio, es importante definir el objetivo o los objetivos que serán rastreados con la estación, es decir, para la definición de la estación se tendrán que especificar los satélites con los cuales la estación tendrá comunicación. Vale la pena recalcar, que la estación será de rastreo de satélites, en este caso se omite el envío de telecomandos al satélite y se establece la comunicación del satélite a la estación [5].

\section{DISEÑO DE LA ESTACIÓN}

Para poder establecer la comunicación con los satélites a ser rastreados, se determinó el punto de referencia donde la estación terrena estará ubicada dentro de las instalaciones de la Fundación Universitaria Los Libertadores en la ciudad de Bogotá D.C., como se ilustra en la Tabla 1.

Tabla 1. Ubicación Estación Terrena de la Fundación Universitaria Los Libertadores

\begin{tabular}{cc}
\hline $\begin{array}{c}\text { Fundación Universitaria Los Libertadores } \\
\text { Laboratorio de Radiometría Solar }\end{array}$ \\
\hline Latitud & $4^{\circ} 39^{\prime} 6.17^{\prime \prime}$ \\
Longitud & W $74^{\circ} 35^{\prime} 6.89^{\prime \prime}$ \\
Altitud & 2579 m.s.n.m \\
\hline
\end{tabular}

A través del software libre de observación satelital Orbitron 3.71 se establecieron los satélites que pasaban por la ubicación de la Tabla 1. A partir de la observación, con el software mencionado, se pudo determinar que seis satélites del tipo CubeSat, universitarios o del segmento radioaficionado podían ser rastreados desde la localización de la Fundación Universitaria Los Libertadores, los datos son presentados en la Tabla 2.

Tabla 2. Satélites para rastrear desde la Estación Terrena de la Fundación Universitaria Los Libertadores

\begin{tabular}{|c|c|c|c|c|c|}
\hline $\begin{array}{c}\text { Nombre } \\
\text { satélite }\end{array}$ & Órbita & Modulación & Frecuencia (MHz) & Polarización & Operativo \\
\hline $\begin{array}{l}\text { SO-50 (Saudi- } \\
\text { Sat 1C- Saudi } \\
\text { OSCAR 50) }\end{array}$ & $\begin{array}{l}593 \times 697 \mathrm{~km}, \\
64,6 \text { grados } \\
\text { de inclinación }\end{array}$ & $x$ & $\begin{array}{c}\text { Subida en VHF }(145.850 \mathrm{MHz}) \\
\text { y bajada en UHF }(436.795 \\
\mathrm{MHz})\end{array}$ & Lineal & Si \\
\hline $\begin{array}{l}\text { FO-29 (JAS-2 } \\
\text { - Fuji OSCAR } \\
\text { 29) }\end{array}$ & $\begin{array}{c}800 \times 1322 \\
\mathrm{~km}\end{array}$ & $\begin{array}{l}\text { Telemetría / baliza: } \\
435.795 \text { MHz (CW) } \\
\text { Digitalker } 435.795 \\
\text { MHz FM (rara vez } \\
\text { operacional) }\end{array}$ & $\begin{array}{c}\text { Subida en VHF (145.900 MHz a } \\
146.000 \mathrm{MHz}) \text {. Bajada en UHF } \\
\text { (435.800 MHz a } 435.900 \mathrm{MHz} \\
\text { - invertido) }\end{array}$ & Circular & Si \\
\hline $\begin{array}{c}\text { AO-73 } \\
\text { (FunCube-1) }\end{array}$ & $\begin{array}{l}\text { sol-síncrona } \\
\text { de } 685 \text { x } 595 \\
\text { k con inclina- } \\
\text { ción de } 97.8 \\
\text { grados }\end{array}$ & $\begin{array}{c}\text { Telemetría / baliza: } \\
145.935 \mathrm{MHz}\end{array}$ & $\begin{array}{c}\text { Subida en UHF (435.130 MHz } \\
\text { a } 435.150 \mathrm{MHz}) \text {. Bajada en } \\
\text { VHF (145.950 MHz a } 145.970 \\
\text { MHz- invertido) }\end{array}$ & Lineal & $\mathrm{Si}$ \\
\hline
\end{tabular}




\begin{tabular}{|c|c|c|c|c|c|}
\hline UBAKUSAT & $\begin{array}{l}400 \mathrm{~km} \mathrm{x} \\
400 \mathrm{~km}\end{array}$ & $\begin{array}{c}\text { Telemetría / bali- } \\
\text { za: } 437.225 \mathrm{MHz} \\
\text { (CW) / telemetría } \\
437.325 \mathrm{MHz}\end{array}$ & $\begin{array}{c}\text { Subida en VHF (145.940 MHz } \\
\text { a } 145.990 \mathrm{MHz}) \text {. Bajada en } \\
\text { UHF (435.200 MHz a } 435.250 \\
\text { MHz). }\end{array}$ & $x$ & Si \\
\hline $\begin{array}{l}\text { NO-44 (PCSat } \\
\text { - Navy OSCAR } \\
44)\end{array}$ & $\begin{array}{l}790 \times 797 \\
\text { km con } 67 \\
\text { grados de } \\
\text { inclinación }\end{array}$ & No tiene & $\begin{array}{c}\text { Subida y bajada en VHF } \\
\text { (145.825 MHz) FM FSK, AX.25, } \\
1200 \text { bps y } 9600 \text { bps. }\end{array}$ & Lineal & SEMI \\
\hline $\begin{array}{l}\text { NO-84 (PSat } \\
\text { New OSCAR } \\
\text { 84) }\end{array}$ & $\begin{array}{l}790 \times 797 \\
\text { km con } 67 \\
\text { grados de } \\
\text { inclinación }\end{array}$ & No tiene & $\begin{array}{c}\text { Subida en HF (28.120 MHz } \\
\text { PSK31 y SSB). Bajada en VHF } \\
\text { ( } 145.825 \mathrm{MHz} \text { Packet } 1200 \\
\text { bps X.25 y APRS - compartido } \\
\text { con ISS, PCSAT y otros). Bajada } \\
\text { en UHF (435.350 MHz PSK31 } \\
\text { FM) }\end{array}$ & Lineal & Si \\
\hline
\end{tabular}

Los satélites seleccionados fueron escogidos por su cercanía, en términos de trayectoria, de la localización de la estación de la Estación Terrena. A partir de los datos de los satélites seleccionados se realizará el presupuesto de comunicaciones de la estación o Link Budget. Es necesario aclarar que con base en la información de estos satélites se determinó el diseño principal de la Estación Terrena; sin embargo, la estación tendrá la capacidad de rastrear otros satélites, dependiendo de sus frecuencias y de sus características de operación.

\section{Link Budget}

El Link Budget o presupuesto de comunicaciones corresponde a la contabilidad de todas las pérdidas y ganancias de un transmisor hacia un receptor en un sistema de telecomunicaciones. Orduy recomienda usar un Presupuesto de enlace de transpondedor analógico básico disponibilizado en Excel1 por Amsat-UK (The Radio Amateur SateIlite Corporation - United Kingdom). La aplicación que se encuentra en Excel define la ganancia de las antenas, la potencia del transmisor y otros parámetros de los componentes. La finalidad del presupuesto es identificar si el enlace es viable entre ellos [5].

Para el propósito del diseño preliminar se establecieron cinco enlaces de comunicación. La Figura 2, presenta los elementos relacionados con la frecuencia del satélite, se observa que la frecuencia de trasmisión y recepción de información corresponde a la banda VHF y es la misma para los dos. Con relación a la longitud de onda de la frecuencia del satélite y la altitud de la órbita la aplicación calcula las perdidas por la propagación.

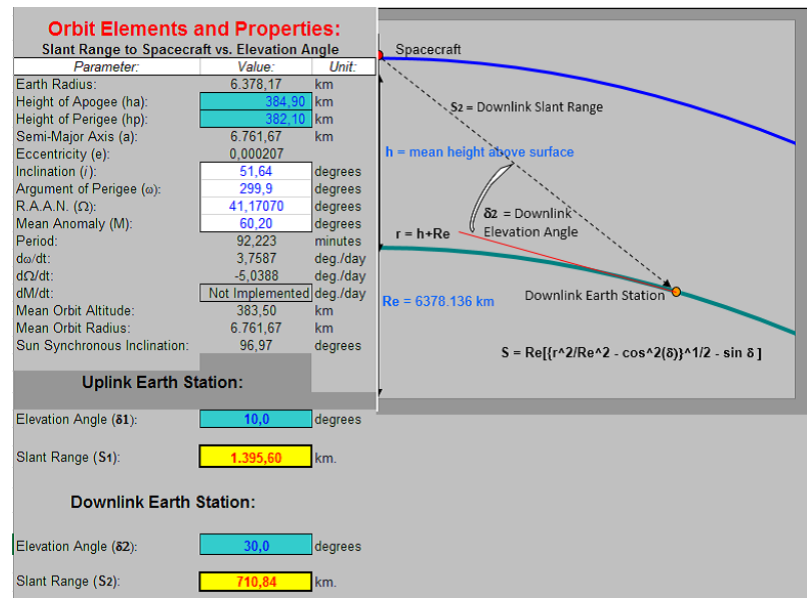

Figura 2. Elementos y propiedades orbitales

Topología de la estación

La topología que se quiere establecer en la Estación portable-Modular de la Fundación Universitaria Los Libertadores, está basada en la estación Terrena PY2SDR (PAR) ya que usa un computador portátil de bajo costo, además, usa la tecnología SDR para rastrear pequeños satélites; el 
software utilizado está disponible por la comunidad internacional de radioaficionados [5]. La Figura 3, presenta la arquitectura que tendrá la estación.

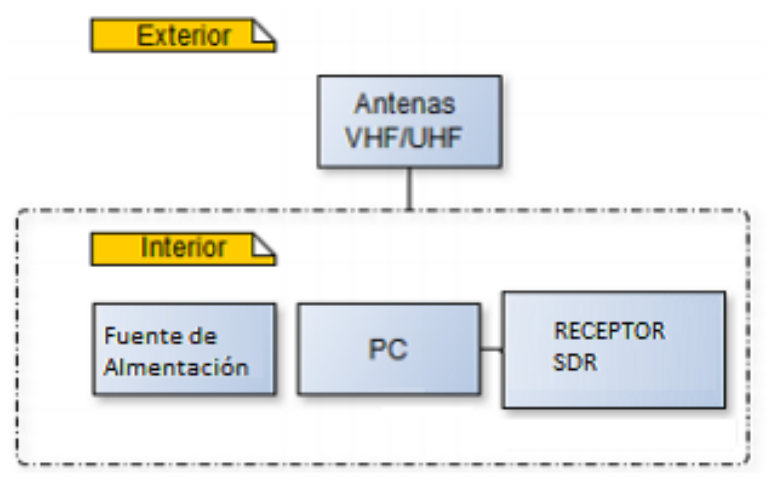

Figura 3. Topología de la Estación Terrena de la Fundación Universitaria Los Libertadores [5].

\section{Sistema irradiante}

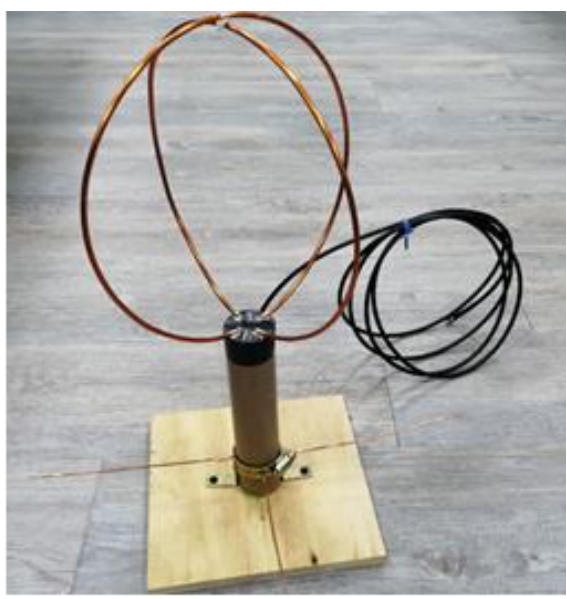

Figura 4. Estructura externa e interna de la Antena UHF

Según la topología de la Estación Terrena para la Fundación Universitaria Los Libertadores, esta debe contar con antenas en las bandas VHF/UHF, sin embargo, manteniendo la idea original de este proyecto, que se basa en bajo costo, se decidió construir parte del hardware de la estación estableciendo una antena del tipo EggBeater en banda UHF que supla las necesidades de la Estación Terrena. Otro factor importante para elaboración del sistema irradiante (antena) fue la necesidad de un sistema compacto debido al entorno local de referencia en donde sería posteriormente ubicada la estación (región montañosa con colinas altas y obstáculos cerca de la estación como edificaciones aledañas a la Fundación Universitaria Los Libertadores).

\section{SDR Comercial (RTL-SDR v.3)}

Según Zabala, Cabeza, Pacheco, Casignia, \& Oñate este dispositivo es un dongle2 USB de bajo costo que se puede usar como un escáner de radio basado en computadora para recibir señales de radio sin necesidad de internet, el cual puede recibir frecuencias desde $500 \mathrm{kHz}$ hasta 1.75 GHz [8]. La Figura 5, muestra el dongle usado en la Estación Terrena.

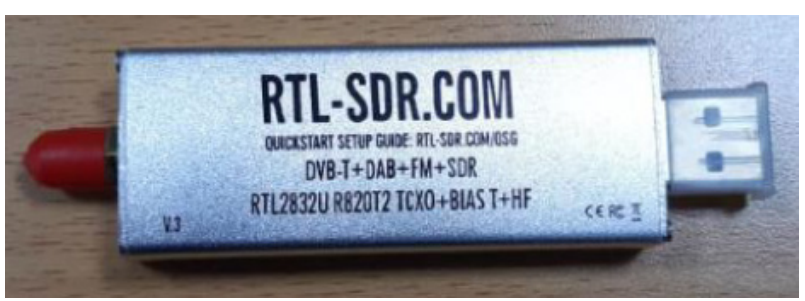

Figura 5. Dongle RTL-SDR

\section{Elementos en software de la Estación}

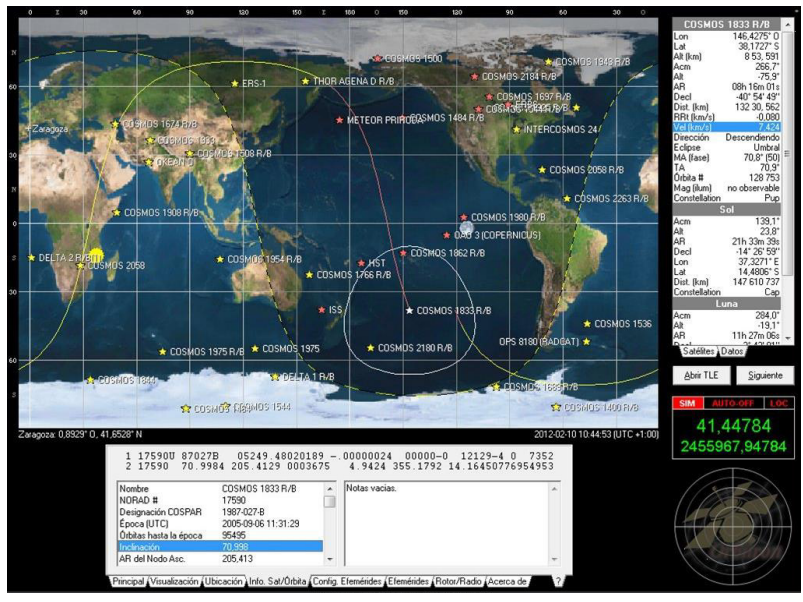

Figura 6. Software de previsión Orbitron

El computador de la Estación Terrena a ser implementada en la Fundación Universitaria Los Libertadores utiliza 
como sistema operativo Microsoft Windows, en este caso se utiliza Microsoft Windows 10. Para realizar el registro de satélites se utiliza el software de previsión Orbitron versión 3.71, como se muestra en la Figura 6.

El software que controla el Dongle RTL-SDR es el SDR SHARP (SDR\#), su propósito general es permitir que el RTL-SDR funcione como un receptor de radio de banda ancha normal, como se muestra en la Figura 6. Por último, vale aclarar que la estación no posee un software de misión específica destinado a decodificar los paquetes de telemetría adquiridos de los satélites específicos, de tal forma, únicamente, se pueden recibir los paquetes y demostrar que está llegando la información.

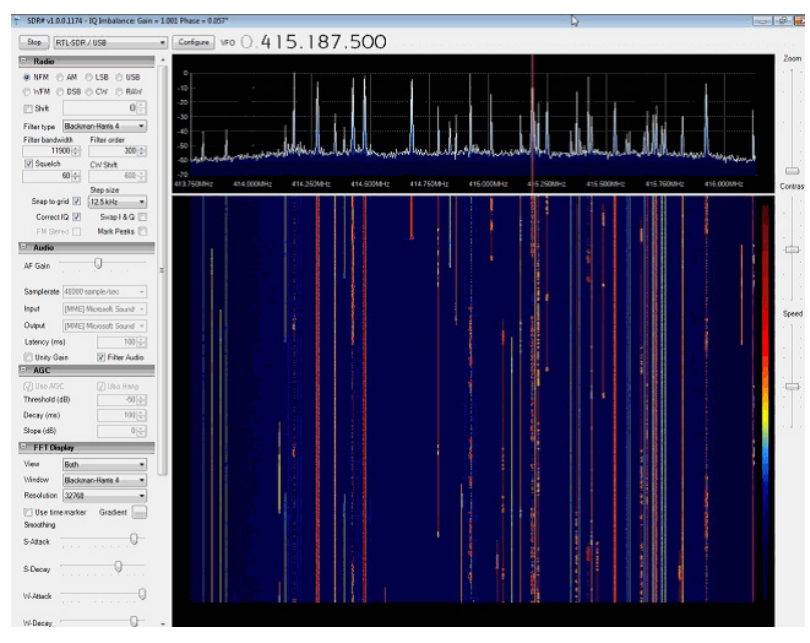

Figura 7. Software de radio SDR SHARP.

\section{RESULTADOS}

Se presentan las especificaciones de la Estación Terrena. La estación Terrena de tipo Portable-Modular a implementar en la Fundación Universitaria Los Libertadores cuenta con un sistema irradiante, un radio del tipo SDR y un Software que localiza los satélites y otro que descarga los paquetes de datos de la señal. El costo total, como se muestra en la Figura 8 es de 282.900 COP (valor de octubre de 2019). De igual forma, se presentan los componentes, su especificación y el fabricante.

\begin{tabular}{|c|c|c|c|c|}
\hline \multirow[b]{2}{*}{ Componente } & \multirow[b]{2}{*}{ Especificación } & \multirow[b]{2}{*}{ Fabricante } & \multicolumn{2}{|c|}{ Costo: } \\
\hline & & & \begin{tabular}{|c|} 
Pesos \\
Colombianos:
\end{tabular} & Dólares $^{4}$ \\
\hline Radio (SDR) & $\begin{array}{l}\text { Rtl-sdr R820t2 Rt|2832u } \\
\text { 1ppm Tcxo Sma Defined }\end{array}$ & $\begin{array}{c}\text { RTL- } \\
\text { SDR.COM }\end{array}$ & 208200 & 61,23 \\
\hline \multirow{6}{*}{$\begin{array}{l}\text { Estructura de la } \\
\text { antena }\end{array}$} & $\begin{array}{c}\text { Tubo de cobre de } 5 \mathrm{~mm}(0.20 \\
\text { in) de diámetro } 136 \mathrm{~cm} \text { de } \\
\text { largo (53.5433). }\end{array}$ & \multirow{8}{*}{ COTS } & 11000 & 3,23 \\
\hline & $\begin{array}{l}\text { Alambre de cobre de } 2 \mathrm{~mm} \\
\text { (0.08 in) de diámetro y } 83 \mathrm{~cm} \\
\text { de largo ( } 32.6772 \mathrm{in}) .\end{array}$ & & 6000 & 1,76 \\
\hline & $\begin{array}{l}\text { Tubo de PVC de } 4.1 \mathrm{~cm}(1.62 \\
\text { in) de diámetro. }\end{array}$ & & 5000 & 1,47 \\
\hline & $\begin{array}{l}\text { Tabla de madera de } 20 \mathrm{~cm} * \\
20 \mathrm{~cm} \text { (Soporte). } 1 \text { unidad. }\end{array}$ & & 2000 & 0,58 \\
\hline & $\begin{array}{c}\text { Len hierro colado de } 3 \mathrm{~cm} * \\
3 \mathrm{~cm} 2 \text { unidades. }\end{array}$ & & 1100 & 0,32 \\
\hline & $\begin{array}{l}\text { Abrazadera cremallera acero } \\
\text { inox, } 32 \mathrm{~mm} \text {. }\end{array}$ & & 600 & 0,17 \\
\hline $\begin{array}{l}\text { Bucles de la } \\
\text { antena }\end{array}$ & Bucles de la antena & & 15000 & 4,41 \\
\hline Línea de fase & $\begin{array}{l}\text { Cable coaxial de longitud de } \\
\text { onda de cuarto de onda RG62 }\end{array}$ & & 34000 & 10 \\
\hline & & TOTAL: & 282.900 & 83,20 \\
\hline
\end{tabular}

Figura 8. Especificaciones de la estación

Finalmente, se presenta la estación Terrena implementada en la terraza del sexto piso de la sede Santander, Figura 9, donde está ubicado el Laboratorio De Radiometría Solar, de la Facultad de Ingeniería y Ciencias Básicas, de la Fundación Universitaria Los Libertadores. La estación se ubicó en un rack prestado por el laboratorio, el cual cuenta con alimentación (110v AC) para alimentar el computador de la estación.

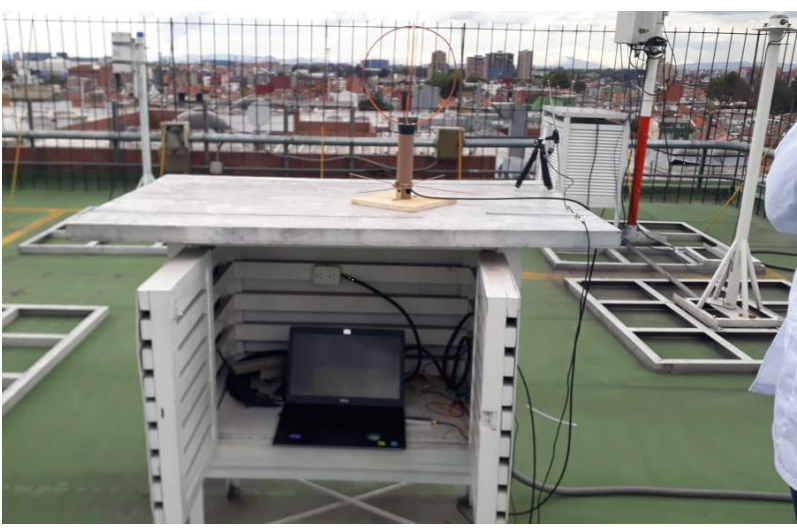

Figura 9. Estación implementada en el Laboratorio de radiometría solar

Luego de haber montado el hardware y software de la estación se procede a recibir información de los satélites 
que fueron seleccionados para realizar el rastreo. Para este fin se estableció una metodología básica para poder recibir datos de los satélites. Es necesario aclarar que la antena opera en la banda UHF (430-450 MHz), es decir, únicamente se podrán recibir datos (Downlink) de satélites en esta banda.

Etapa 1. En primer lugar, se debe recolectar la información de los satélites, esto se puede hacer en internet desde una página web que especifique las frecuencias de operación. La Figura 10, muestra las frecuencias Downlink y Beacon del satélite BRICSAT 2 (NO-103).

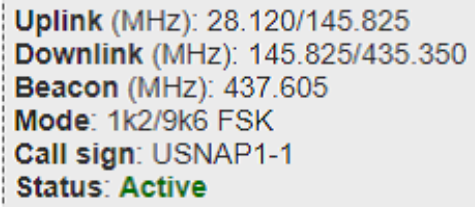

Figura 10. Frecuencias de operación del BRICSAT 2 (NO-103)

Etapa 2. Luego de haber de establecido los parámetros del satélite se debe accionar el programa Orbitron, el cual mostrará la pasada del satélite sobre la estación. En el momento que el software muestre que el satélite esté encima de la estación se podrá realizar el rastreo del satélite y la descarga de datos. La Figura 11, muestra el satélite BRICSAT 2 (NO-103), sobre la estación de la Fundación Universitaria Los Libertadores.

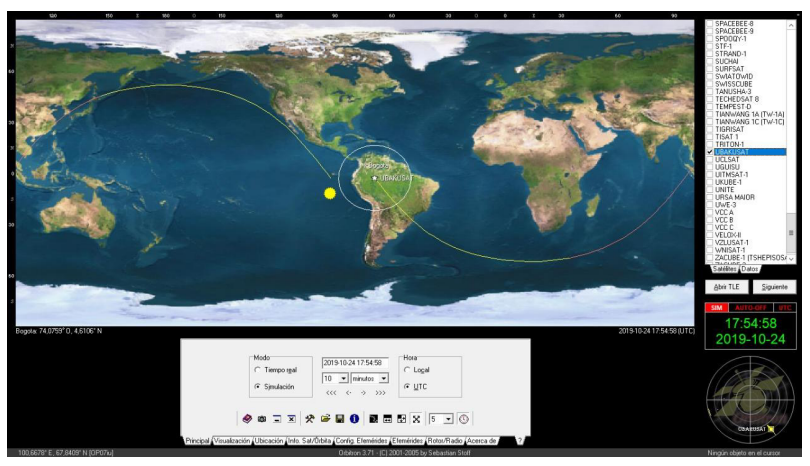

Figura 11. Software Orbitron - Pasada del satélite
Etapa 3. Una vez determinada la frecuencia de Downlink del satélite y de saber la posición de este, se procede a accionar el software de radio SDR\#, el cual mostrará la señal que se esté recibiendo. Se pudo establecer el rastreo a un satélite, ya que con los otros satélites no se especificó si las señales llegaban o eran propias de esos satélites, en la Figura 12 se presenta el Software SDR\# recibiendo el Beacon del satélite BRICSAT 2 (NO-103) se puede establecer que se tiene que hacer una corrección de la frecuencia debido al efecto doppler, ya que la frecuencia del Beacon era $437.605 \mathrm{MHz}$ y se recibieron datos del Beacon en $441.913 \mathrm{MHz}$.

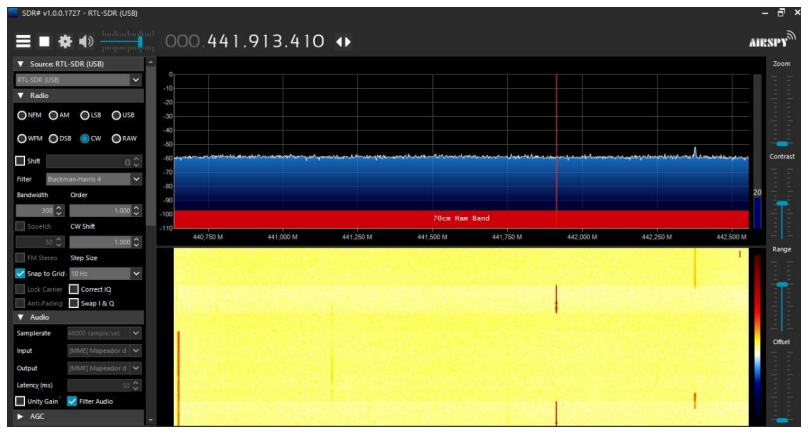

Figura 12. Software SDR\# - Beacon del satélite BRICSAT 2 (NO-103)

\section{DISCUSIÓN}

Como referencia, y como se mencionó anteriormente, se tomó la estación terrena PY2SD, la cual está basada en un diseño de radioafionado simple pero funcional. La estación es fija de bajo costo que tuvo como principio usar tecnología SDR, para de este modo poder desempeñar sus funciones utilizando Software y Hardware comercial puesto a disposición por la comunidad radioaficionada internacional. La estación PY2SD posee cuatro elementos en Hardware: un computador, un SDR, un Radio analógico y una antena.

Por otro lado, cuenta con un software que controlador del SDR (Software SDR\# v1.0.0.1357) y otro software de apoyo para telemetría satelital (AMSAT-BR Plan73, Fox Telemetry Analysis Tool) desarrollado por el propietario. 
El costo aproximado de elaboración de la estación es de USD \$1,200 (valor de octubre de 2015) .

Con relación a la estación PY2SDR, la Estación Terrena FULL cumple el diseño de radioaficionado simple, pero funcional. La estación FULL posee un elemento menos en Hardware: el Radio analógico, ya que como es una estación de rastreo no es necesario el envío de telecomandos. Por otro lado, comparando el software, la estación FULL posee una versión mejorada del software de del controlador del SDR (SDR\# v3) y otro software de rastreo de satélites Orbitron; sin embargo, no cuenta con un software de decodificación de datos el cual se vuelve indispensable a la hora de conocer la información del satélite. La estación FULL equivale a menos del $23 \%$ del costo de elaboración de esta estación.

\section{CONCLUSIONES}

El diseño de estación terrena que fue implementado en la Fundación Universitaria Los Libertadores cuenta con tres elementos en Hardware: una Antena, un Radio SDR y un Computador. El Software y el diseño que fue implementado en la Fundación Universitaria Los Libertadores cuenta con tres elementos, a saber: Sistema operativo WINDOWS 10, Controlador del SDR, SDR\# V3 y para el rastreo de Satélites se utilizó el software libre ORBITRON. El costo aproximado de elaboración de la estación FULL fue de \$ 287.20 USD (valor de octubre de 2019).

Finalmente, se pudo establecer que con un bajo presupuesto y utilizando componentes COTS adquiridos en mercado sin mayor problema, se puede desarrollar un sistema con filosofía modular y flexibilidad de operación, ya que se puede adaptar para conseguir la comunicación con varios satélites.

Es importante destacar que el proyecto de la estación terrena fue el resultado de la implementación del espacio académico Tecnología de Sistemas Satelitales en el programa de Ingeniería Aeronáutica de la Facultad de Ingeniería y Ciencias Básicas de la Fundación Universitaria los Libertadores.

\section{REFERENCIAS}

[1] F. A Osorio and E. R. Andrade. Sistemas satelitales (Bachelor's thesis).Ecuador. 2006, pp. 15.

[2] D. Ilchikawa. Cubesat-to-ground communication and mobile modular ground station development. honolulu, hawaii: university if hawaii at manoa. 2006, 6 p.

[3] O. penna. Instalación y operación de una estación terrena para satélites de órbita baja. Facultad de Ingeniería, Universidad Nacional Autónoma de México, México. D.F. 2011, 176p.

[4] A. Wickramanayake. Design, development and operation of a student ground Station. Lulea University of Technology, Department of Space Science, Kiruna, Lulea, Suecia, 2007, 76p.

[5] J. Orduy, Processo De Referência Para O Desenvolvimento Da Arquitetura De Uma Estação Terrena Para Pico E Nanosatélites". Instituto Nacional de Pesquisas Espaciais. Brasil, 2016.

[6] J. Lozada. Investigación aplicada: Definición, propiedad intelectual e indústria. CienciAmérica: Revista de divulgación científica de la Universidad. Tecnológica Indoamérica. 2014, 47-50.

[7] E. J. Chikofsky and J. H. Cross. Reverse engineering and design recovery: A taxonomy. IEEE software, 7(1). 1990, 13-17.

[8] M. Zabala, G. Cabeza, M. Pacheco, B. Casignia and A. Oñate. Sistema de recepción de información satelital basado en SDR. Ciencia Digital, 2019, 446-463. 